\title{
Experimental infection of dairy calves with Ehrlichia chaffeensis
}

Jose R. C. delos Santos, ${ }^{1}$ Kirsten Boughan, ${ }^{1}$ William G. Bremer, ${ }^{1}$ Brian Rizzo, ${ }^{1}$ John J. Schaefer, ${ }^{2}$ Yasuko Rikihisa, ${ }^{3}$ Glen R. Needham, ${ }^{2}$ L. A. Capitini, ${ }^{4}$ David E. Anderson, ${ }^{5}$ Michael Oglesbee, ${ }^{3}$ S. A. Ewing ${ }^{6}$ and Roger W. Stich

Correspondence

Roger W. Stich

stichrw@missouri.edu
Received 2 June 2007

Accepted 27 July 2007

\author{
${ }^{1}$ Department of Veterinary Preventive Medicine, Ohio State University, Columbus, OH, USA \\ ${ }^{2}$ Department of Entomology, Ohio State University, Columbus, OH, USA \\ ${ }^{3}$ Department of Veterinary Biosciences, Ohio State University, Columbus, OH, USA \\ ${ }^{4}$ University Laboratory Animal Resources, Ohio State University, Columbus, OH, USA \\ ${ }^{5}$ Department of Veterinary Clinical Sciences, Ohio State University, Columbus, OH, USA \\ ${ }^{6}$ Department of Veterinary Pathobiology, Oklahoma State University, Stillwater, OK, USA \\ ${ }^{7}$ Department of Veterinary Pathobiology, University of Missouri, Columbia, MO, USA
}

\section{INTRODUCTION}

Ehrlichia chaffeensis is an obligate intracellular bacterial parasite and is the aetiologic agent of human monocytic ehrlichiosis (HME), an important emerging tick-borne infectious disease. Clinical outcomes associated with this disease range from asymptomatic to severe and even fatal (Eng et al., 1990b; Everett et al., 1994; Fishbein et al., 1994). E. chaffeensis appears to cause unapparent or mild clinical manifestations in non-primate hosts and this, together with challenges in detecting the pathogen, makes the experimental characterization of $E$. chaffeensis transmission

Abbreviations: d.p.i., days post-inoculation; HME, human monocytic ehrlichiosis; IFA, immunofluorescence assay; i.v., intravenous(ly); WBC, white blood cell. and pathogenesis difficult. A reliable E. chaffeensis-based animal model of HME would facilitate the understanding of the transmission, pathology and immunology of this malady (Dawson \& Ewing, 1992; Dawson et al., 1994; Ewing et al., 1995; Lockhart \& Davidson, 1999; Unver et al., 2002; Zhang et al., 2003; Dugan et al., 2004; Loftis et al., 2004).

A wide range of mammals are reported to be naturally infected with E. chaffeensis, including white-tailed deer (Dawson et al., 1994; Ewing et al., 1995; Davidson et al., 2001; Varela et al., 2003), domestic goats (Dugan et al., 2000), domestic and wild canids (Dawson et al., 1996; Davidson et al., 1999), and lemurs (Williams et al., 2002). Cattle are domestic hosts of the ticks Amblyomma americanum (Barnard, 1988; Kollars et al., 2000) and 
Dermacentor variabilis (Bishopp \& Tremblet, 1945; Smith et al., 1946; Lancaster, 1973); both A. americanum and D. variabilis are reportedly infected with $E$. chaffeensis in nature (Eng et al., 1990a; Anderson et al., 1993; Ewing et al., 1995; Roland et al., 1998; Kramer et al., 1999), and thus cattle are likely to be exposed to E. chaffeensis under natural conditions.

Despite investigations that suggest that $E$. chaffeensis and $A$. americanum are both adapted to a variety of mammals, and the potential importance of bovine host infection from epidemiological, economic and experimental standpoints, the susceptibility of cattle to infection with E. chaffeensis has not been reported to date. We investigated this susceptibility by inoculating dairy calves with DH82 cells infected with the Arkansas, St Vincent or 91HE17 strain of E. chaffeensis. In the first experiment, splenectomized and spleen-intact calves were injected with thawed stabilates of E. chaffeensis-infected DH82 cells. In the second experiment, spleen-intact calves were injected with active cultures of DH82 cells infected with the same E. chaffeensis strains. Calves in both experiments were monitored for signs of infection with E. chaffeensis, including xenodiagnosis by assay of $A$. americanum and $D$. variabilis ticks allowed to feed as nymphs on the inoculated calves.

\section{METHODS}

E. chaffeensis. Arkansas, St Vincent and 91HE17 isolates were cultivated in a DH82 cell line, as previously reported (Rikihisa et al., 1991, 1994; Wen et al., 1997). Dr David Walker of the University of Texas Medical Branch at Galveston graciously provided the 91HE17 isolate. The Arkansas isolate was supplied from within the Ohio State University Department of Veterinary Biosciences. The E. chaffeensis St Vincent isolate (ATCC no. VR-1454) was purchased from the ATCC.

For the first experiment, the three isolates were prepared so that the relative number of organisms could be standardized for each inoculum. Real-time PCR was adapted from a previously reported PCR assay to determine relative $p 28$ copy numbers for the cryopreserved E. chaffeensis-infected DH82 cell stabilates (Wagner et al., 2004). This assay is specific for a single omp-1 paralogue; therefore, a single copy of the target amplicon was expected for each genome. The $p 28$ amplicon was cloned into the pCR2.1-TOPO TA vector (Invitrogen) according to the manufacturer's instructions, and these plasmids were used for standardization of organism numbers in the different strain stabilates. Stabilate aliquots were overlaid with mineral oil and heated to $105{ }^{\circ} \mathrm{C}$ for $15 \mathrm{~min}$, cooled on ice for $>1 \mathrm{~min}$ and assayed at $1 \times 10^{0}, 10^{-1}$ and $10^{-2}$ dilutions. Quantitative real-time PCR was performed with the iQ SYBR Green Supermix (Bio-Rad) and an iCycler (Bio-Rad) at the Ohio State University Plant Microbe Genome Facility. Reaction profiles consisted of an activation step at $95{ }^{\circ} \mathrm{C}$ for $15 \mathrm{~min}$, followed by 40 cycles of $94{ }^{\circ} \mathrm{C}$ (1 min), $60{ }^{\circ} \mathrm{C}(1 \mathrm{~min})$ and $72{ }^{\circ} \mathrm{C}(1 \mathrm{~min})$ for denaturation, annealing and extension steps, respectively. Standard curves were used to calculate $p 28$ copy numbers for each isolate, which were then adjusted to $1.5 \times 10^{8}$ copies in the context of $5 \times 10^{6} \mathrm{DH} 82$ host cells per inoculum.

For the second experiment, active cultures of each strain were harvested and pooled, and DH82 cells were counted on a haemocytometer, while the percentages of infected DH82 cells were calculated with stained cytospin preparations. Each inoculum was adjusted to $2.0 \times 10^{7}$ DH82 cells, $80 \%$ of which contained $E$. chaffeensis morulae, except for the negative control doses that consisted of $2.0 \times 10^{7}$ non-infected DH82 cells.

Experimental cattle. Calves were cared for in accordance with a protocol approved by and on file with the Ohio State University Institutional Laboratory Animal Care and Use Committee. These calves were maintained on antibiotic-free feed, and provided with hay and water ad libitum. The calves were pre-screened for exposure to $E$. chaffeensis by PCR assay of buffy coats and immunofluorescence assay (IFA) of plasma. Venous blood was collected with EDTA for PCR, IFA and haematology. Complete blood counts were performed at the Ohio State University College of Veterinary Medicine clinical laboratory with a Cell-Dyne 3500 cytometer (Abbott Laboratories). Baseline data for body temperature, packed cell volume, platelet count, white blood cell (WBC) count and differential leukocyte count were obtained prior to exposure to E. chaffeensis.

In the first experiment, seven bull calves were obtained from a local livestock auction market. These calves were $2-3$ months old and of various dairy breeds, in addition to Holstein, and weighed between 72 and $158 \mathrm{~kg}$ at the start of the study. Four of these animals were splenectomized, one of which served as a negative control. The surgery was performed at the Ohio State University Large Animal Surgery Unit of the Department of Clinical Sciences, College of Veterinary Medicine. The splenectomized calves were inoculated 3 weeks after surgery. The three remaining calves were left spleenintact. Pairs of normal and splenectomized calves were inoculated intravenously (i.v.) with different E. chaffeensis strains (Arkansas, St Vincent or 91HE17) that were cryopreserved as frozen stabilates.

In the second experiment, eight 2-month-old Holstein bull calves, with weights ranging from 90 to $130 \mathrm{~kg}$, were obtained from a local dairy farm. The same E. chaffeensis strains were used for this experiment, except that the organisms were actively growing in DH82 cells immediately prior to inoculation (i.v.) into two calves for each strain. A fourth pair of calves was inoculated with uninfected DH82 cells to serve as a negative control.

Analysis of clinical data. Body temperatures and complete blood counts were recorded for a minimum of 2 weeks prior to inoculation. These baseline data were used to transform quantitative clinical parameters into $Z$ scores by subtraction of the baseline mean value for each calf from each post-inoculation observation and division of this difference by the respective baseline SD. $Z$ scores for each observation and parameter were plotted together for each host.

Xenodiagnosis. A. americanum and D. variabilis nymphs were purchased from the Oklahoma State University Medical Entomology Laboratory. Nymphs of each species were fed to repletion on each calf, as described elsewhere (Stich et al., 1989). These ticks were placed on the calves at 31 (experiment 1 ) and 28 (experiment 2) days postinoculation (d.p.i.) with E. chaffeensis. Replete nymphs were held in an environmental chamber (room temperature, 95-100\% relative humidity, $12: 12 \mathrm{~h}$ light:dark photoperiod) and DNA was isolated after they moulted into the adult stage.

PCR assay. Bovine blood was collected with EDTA, and buffy coats were isolated after centrifugation at $910 \mathrm{~g}$ for $30 \mathrm{~min}$. Residual erythrocytes were lysed with two volumes of TE $(10 \mathrm{mM}$ Tris- $\mathrm{HCl}$, $\mathrm{pH}$ 8.0, $1 \mathrm{mM}$ EDTA), followed by centrifugation at $14000 \mathrm{~g}$ for $5 \mathrm{~min}$ and resuspension in TE for a total of three washes. The final cell suspension was in $500 \mu \mathrm{l}$ protein digestion buffer $(100 \mathrm{mM}$ Tris, $\mathrm{pH}$ 8.0, $1 \mathrm{mM}$ EDTA, $2 \%$ SDS, $0.1 \mathrm{mg}$ proteinase $\mathrm{K} \mathrm{ml}^{-1}$ ), which was then incubated overnight at $55{ }^{\circ} \mathrm{C}$. Digests were extracted once each with equal volumes of buffer-saturated phenol $(\mathrm{pH}>7.5)$ (Invitrogen Life Technologies), phenol/chloroform/isoamyl alcohol (25:24:1), and chloroform/isoamyl alcohol (24:1). DNA was 
precipitated with 0.1 volume of $3 \mathrm{M}$ sodium acetate and 2.5 volumes of absolute ethanol. The DNA samples were centrifuged at $14000 \mathrm{~g}$ for $15 \mathrm{~min}$ at $4{ }^{\circ} \mathrm{C}$, dried in a vacuum centrifuge, and dissolved in $50 \mu \mathrm{l}$ TE.

Ticks were prepared for DNA isolation by incubation for $80 \mathrm{~h}$ at $37{ }^{\circ} \mathrm{C}$ in $95-100 \%$ relative humidity, bisected along the median plane under aseptic conditions, and digested for $3 \mathrm{~h}$ at $55^{\circ} \mathrm{C}$ in $100 \mu \mathrm{l}$ protein digestion buffer. These digests were subjected to protein extraction and DNA precipitation as described for buffy coat samples, and dissolved in $25 \mu \mathrm{TE}$.

A 16S rDNA-based real-time PCR assay with a fluorogenic probe was used to assay buffy coats and ticks, as described elsewhere (Loftis et al., 2003), except that ABsolute QPCR mix (ABgene) and a Taqmanbased black hole quencher (BHQ) probe (Biosearch) were utilized with an ABI 7300 thermocycler (ABI).

IFA. E. chaffeensis-infected DH82 cells (Arkansas strain) were concentrated to $1.0 \times 10^{6}$ cells ml $^{-1}$ in PBS, placed on Teflon-coated slides (10 $\mu \mathrm{l}$ per well) (Electron Microscopy Sciences), and allowed to air-dry overnight. Dried slides were placed in ice-cold methanol for $5 \mathrm{~min}$ and stored at $-20{ }^{\circ} \mathrm{C}$. Plasma was diluted $1: 20$ for initial screenings, and $10 \mu \mathrm{l}$ per well of each diluted plasma sample was incubated at $37{ }^{\circ} \mathrm{C}$ for $30 \mathrm{~min}$ before rinsing with PBS. The slides were incubated at $37{ }^{\circ} \mathrm{C}$ for $30 \mathrm{~min}$ with $10 \mu \mathrm{l}$ of FITC-conjugated goat anti-bovine $\operatorname{IgG}_{1,2}$ polyclonal antiserum (VMRD) and rinsed with PBS, then mounted with ProLong Antifade reagent (Molecular Probes), according to the manufacturer's instructions. An Olympus BX51 fluorescence microscope with filter cube \#11001v2 (Chroma Technology) was used to view the slides. Titres were determined for samples that tested IFA-positive during the initial screening.

\section{RESULTS AND DISCUSSION}

\section{Inoculation of calves with cryopreserved $E$. chaffeensis}

Several factors were considered prior to attempting experimental infection of calves with $E$. chaffeensis. First, host specificities of different E. chaffeensis strains were uncertain. For example, although there is evidence that goats can be naturally infected with E. chaffeensis, attempted experimental infections of this host species with E. chaffeensis isolated from a human being (Arkansas) and a white-tailed deer (15B-WTD-GA) have been unsuccessful (Dugan et al., 2000, 2004). An E. chaffeensis strain isolated from cattle was not available; therefore, we used three different strains isolated from human beings to inoculate calves. Several E. chaffeensis strains have been isolated from human beings, and fall into at least three major genogroups based on comparison of the locus for the outer-membrane protein-1 (omp-1) paralogue p28 (Yu et al., 1999a; Cheng et al., 2003). The isolates chosen for this study were representative of each of those three groups. The second issue concerned the possible spectrum of virulence among these pathogens (Paddock et al., 2001), which indicated that standardization of experimental doses would be advisable. Initially, we addressed this matter with quantitative PCR of cryopreserved stabilates prepared for each strain. This approach allowed us to calculate copy number and adjust the dose so that each group of calves received comparable amounts of pathogen and host (DH82) cells. Third, the influence of the vertebrate immune system in determining susceptibility to experimental E. chaffeensis infection and the resulting disease was considered. Splenectomized animals have exhibited overt clinical symptoms of disease in instances of latent protozoan and bacterial infections (Ellis \& Smith, 1966; Garnham, 1970). In calves, splenectomy reduces natural resistance to disease caused by haemoparasites such as Anaplasma and Babesia species, resulting in severe disease among challenged animals which is otherwise restricted to older animals (De Kock \& Quinlan, 1926; Roby et al., 1961; Jones \& Brock, 1966). Conversely, more pronounced signs have been reported for dogs that were left spleen-intact than for those that were splenectomized prior to experimental infection with Ehrlichia canis, a species that is closely related to E. chaffeensis (Harrus et al., 1998). Therefore, similarities between the disease susceptibility of splenectomized calves and that of older cattle, coupled with epidemiological implications that age could be a risk factor for severe HME (Roland et al., 1995; Olano et al., 2003a, b; Stone et al., 2004; Demma et al., 2005) and the reportedly greater susceptibility of spleen-intact dogs to canine monocytic ehrlichiosis, compelled us to test both splenectomized and normal calves in the first experiment.

Clinical observations. Mild clinical signs were seen on occasion after inoculation (Fig. 1). A16, a spleen-intact calf inoculated with the 91HE17 strain, exhibited a spike in body temperature $\left(41.1{ }^{\circ} \mathrm{C}\right)$ at 10 d.p.i. Decreases in peripheral leukocytes were indicated for calf A16 on several different dates throughout the study, including during the fever observed at 10 d.p.i. A significant drop in body temperature was observed for A16 after 30 d.p.i. Calves A12, A13 and A14 showed drops in peripheral platelets at about 2 weeks post-inoculation. A12 first experienced a decreased platelet count on 17 d.p.i., with a nadir at 29 d.p.i. A13 and A14 had minimum values at 15 and 13 d.p.i., respectively. A17, a splenectomized control calf inoculated with non-infected DH82 cells, also experienced a decreased platelet count with a nadir at 29 d.p.i.

We did not observe evidence of exacerbation of susceptibility to infection or disease with low-dose inoculations of different E. chaffeensis strains due to splenectomy. Indeed, more pronounced signs were observed among spleen-intact hosts, which corroborated the aforementioned report of experimental E. canis infections among normal and splenectomized dogs (Harrus et al., 1998). Some susceptibility to infection by $E$. chaffeensis, however, was discernible by changes in haematological features of each animal. A close comparison of haematological parameters before and after inoculation suggested these subtle changes. The fever observed for calf A16 at 10 d.p.i. was consistent with the 10 day prepatent period estimated for HME (Everett et al., 1994), and haematological abnormalities such as decreased WBC in A16 and lowered platelet counts 

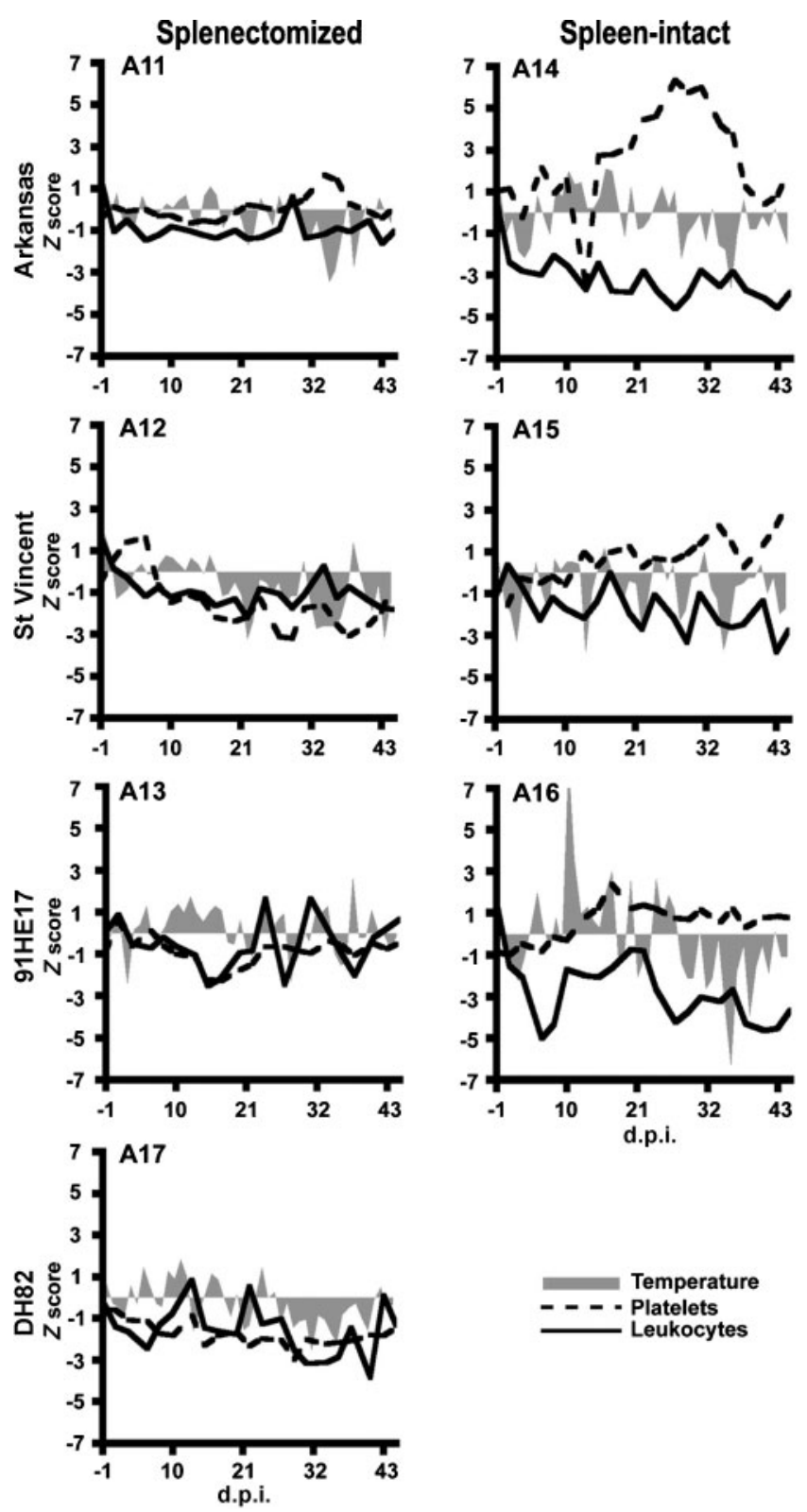

Fig. 1. Clinical observations of calves after inoculation with cryopreserved stabilates of different $E$. chaffeensis strains. $Z$ scores were calculated and plotted for body temperatures (shaded areas), peripheral platelets (dashed lines) and leukocytes (solid lines), as described in the text. Left and right columns represent splenectomized and normal hosts, respectively. The top, second and third rows represent calves inoculated with the Arkansas, St Vincent, and $91 \mathrm{HE} 17$ strains, respectively. The bottom-left panel represents the negative control.

in A12 and A13 were also consistent with the blood picture in humans and dogs (Fishbein et al., 1994; Zhang et al., 2003).

IFA. All calves inoculated with E. chaffeensis-infected DH82 cells tested IFA-positive for antibodies to intracellular
Table 1. Weekly IFA titres of plasma from calves inoculated with cryopreserved stabilate

\begin{tabular}{|c|c|c|c|c|c|c|c|c|}
\hline \multirow[t]{2}{*}{ Inoculum } & \multirow[t]{2}{*}{ Calf } & \multicolumn{7}{|c|}{ d.p.i. } \\
\hline & & 0 & 7 & 14 & 21 & 28 & 35 & 42 \\
\hline DH82 & $\mathrm{A} 17^{\star}$ & $-\dagger$ & - & - & - & - & - & - \\
\hline \multirow[t]{2}{*}{ Arkansas } & $\mathrm{A} 11^{*}$ & - & 20 & 64 & 64 & 64 & 64 & 64 \\
\hline & $\mathrm{A} 14 \ddagger$ & - & 128 & 128 & 128 & 64 & 64 & 64 \\
\hline \multirow[t]{2}{*}{ St Vincent } & $\mathrm{A} 12^{*}$ & - & 64 & 64 & 64 & 64 & 64 & 64 \\
\hline & A15 & - & 64 & 64 & 64 & 64 & 64 & 64 \\
\hline \multirow[t]{2}{*}{$91 \mathrm{HE} 17$} & $\mathrm{~A} 13^{*}$ & - & 64 & 64 & 64 & 64 & 64 & 64 \\
\hline & A16‡ & - & 256 & 512 & 64 & 128 & 64 & 64 \\
\hline
\end{tabular}

${ }^{\star}$ Splenectomized.

$\dagger$ Negative at $1: 20$ dilution.

$\ddagger$ Spleen-intact.

morulae (Table 1). The titres ranged from 20 (calf A11 at 7 d.p.i.) to 512 (A16 at 14 d.p.i.). Immunofluorescent host cells, but not morulae, were observed with plasma from the negative control host, A17. Neither calf inoculated with the Arkansas strain developed an IFA titre greater than 64 . Only two of the remaining calves (A14 and A16) had peak antibody titres greater than 64; the spleen was intact in both of these calves.

PCR assay of peripheral blood. PCR assays of buffy coats yielded positive results for calves A13 and A16 at 25 d.p.i. Both of these calves were injected with the 91HE17 strain. All other buffy coat samples tested during this experiment were PCR-negative.

Xenodiagnosis. Although none of the bovine buffy coats was found to be PCR-positive during the interval when nymphal ticks were acquisition-feeding, the $16 \mathrm{~S}$ rDNAbased real-time PCR assay yielded positive results for each calf when male $D$. variabilis and A. americanum were tested (Table 2).

\section{Inoculation of calves with active cultures of $E$. chaffeensis}

Although signs of infection were observed in the first experiment, results were equivocal. These findings might have resulted from utilization of cryopreserved stabilates that had fewer viable organisms than would be calculated based on $p 28$ copy numbers. The parasites might have been attenuated as a result of cryopreservation, and a potential combination of lower dose and an immunostimulatory effect of attenuated or dead parasites could have affected the clinical outcomes observed. Mindful of this possible impact in experiment 1, E. chaffeensis strains actively growing in DH82 cells were used for the second experiment. The same approach has been used in earlier infection experiments by several investigators (Dawson \& Ewing, 1992; Ewing et al., 1995; Felek et al., 2001; Varela 
Table 2. PCR-positive male ticks that fed as nymphs on calves inoculated with cryopreserved stabilate (from a total of 10 per host)

\begin{tabular}{|lccc|}
\hline \multirow{2}{*}{$\begin{array}{l}\text { E. chaffeensis } \\
\text { strain }\end{array}$} & Calf & \multicolumn{2}{c|}{ Tick species } \\
\cline { 3 - 4 } & & $\begin{array}{c}\text { A. americanum } \\
(\boldsymbol{n}=\mathbf{1 0})\end{array}$ & $\begin{array}{c}\text { D. variabilis } \\
(\boldsymbol{n}=\mathbf{1 0})\end{array}$ \\
\hline \multirow{2}{*}{ Arkansas } & & 3 & 8 \\
& $\mathrm{~A} 11^{\star}$ & 4 & 2 \\
St Vincent & $\mathrm{A} 14 \dagger$ & 7 & 5 \\
& $\mathrm{~A} 12^{\star}$ & 7 & 6 \\
91 HE17 & $\mathrm{A} 15 \dagger$ & 9 & 2 \\
& $\mathrm{~A} 13^{*}$ & 7 & 6 \\
& $\mathrm{~A} 16 \dagger$ & & \\
\hline
\end{tabular}

${ }^{\star}$ Splenectomized.

$\dagger$ Spleen-intact.

et al., 2003). All experimental calves used for the second experiment were spleen-intact.

Clinical observations. Calves A33, A36 and A38, which were inoculated with Arkansas, St Vincent and 91HE17 strains, respectively, became lethargic and developed progressive muscular weakness in their hind limbs. These calves were unable to rise, became sternally recumbent, and eventually died 6-13 d.p.i. All three calves experienced similar symptoms (Fig. 2). A36 and A33 developed a lateral curvature in the neck a few hours before death. These clinical signs became obvious in A36 on the day of its death, 6 d.p.i. The same signs became apparent in A33 at 7 d.p.i., and that calf was euthanized as per the animal use protocol. A38 died at 13 d.p.i., after developing similar symptoms but without the lateral cervical twist. All three calves appeared reluctant to drink water but were observed eating hay while sternally recumbent.

Sharply decreased body temperatures were observed immediately prior to the deaths of calves A36, A33 and A38 (Fig. 2). Calves A34 and A38 had fevers that peaked at $39.5^{\circ} \mathrm{C}$ (4 d.p.i.) and $40.1{ }^{\circ} \mathrm{C}$ (7 d.p.i.), respectively. A36, which was injected with 91HE17, had a sharp increase in peripheral platelets at 1 d.p.i., followed by a simultaneous spike in leukocyte numbers and a sharp decline in platelets at 3 d.p.i.

The resultant morbidity and subsequent mortality among three of the host animals in this experiment was unexpected, and is of interest because, to our knowledge, this is the first report of mortality resulting from experimental infection of vertebrates with $E$. chaffeensis. The generalized muscular weakness and lethargy exhibited prior to death of calves A36, A33 and A38 were reminiscent of clinical features observed with severe HME (Fishbein et al., 1994; Williams et al., 2002). Progressive ataxia leading to inability to stand has also been reported in a mature male dog with dual-species ehrlichial infection
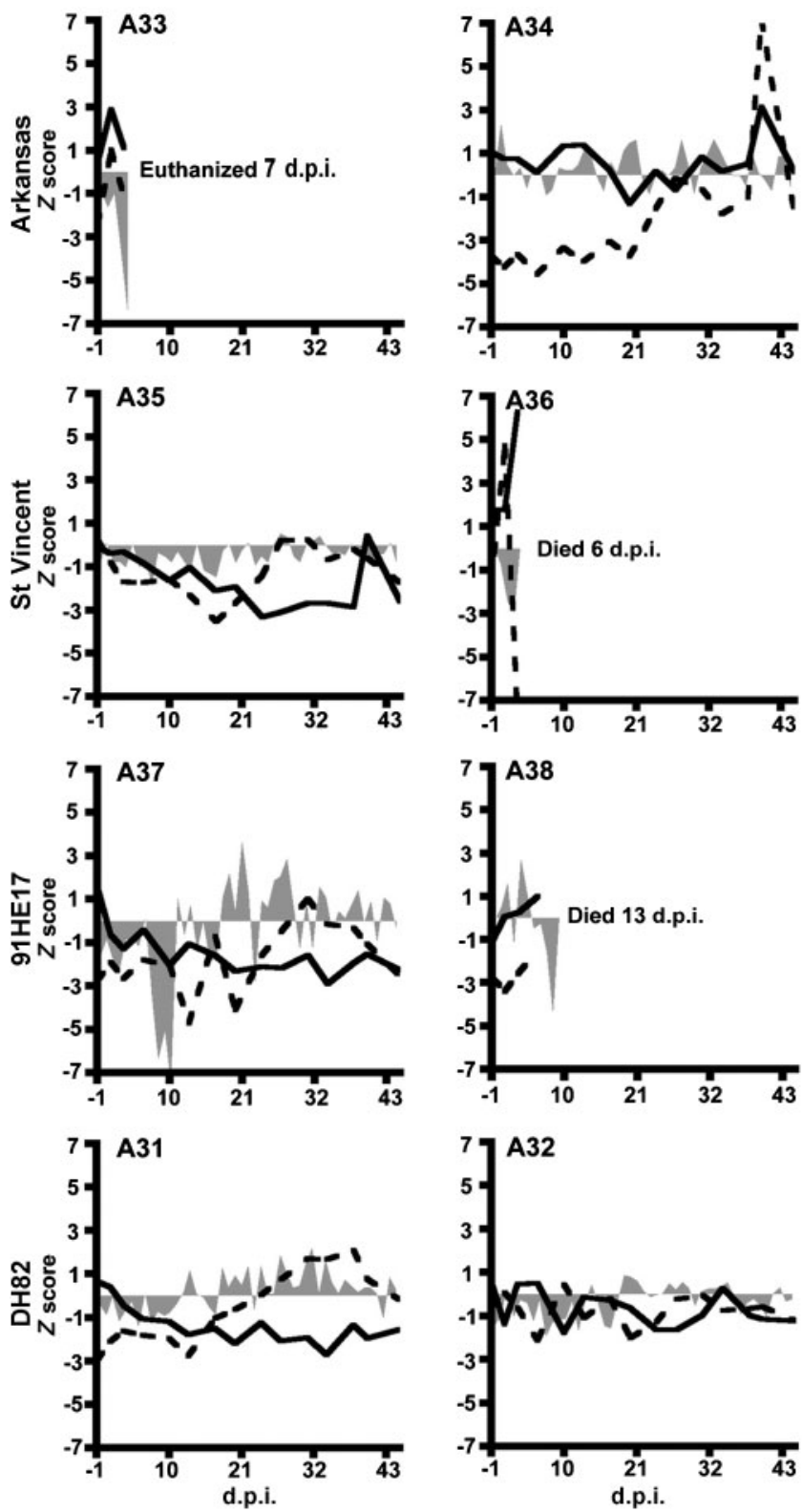

Fig. 2. Clinical observations of calves after inoculation with active cultures of different $E$. chaffeensis strains. $Z$ scores were calculated and plotted for body temperatures (shaded areas), peripheral platelets (dashed lines) and leukocytes (solid lines), as described in the text. Top, second and third rows represent calves inoculated with the Arkansas, St Vincent and 91HE17 strains, respectively. The bottom row represents negative controls.

(Meinkoth et al., 1998). At physical examination, abnormalities noted in this dog included evidence of cervical pain in addition to generalized weakness. The lateral twist of the neck in A36 and A33 suggested a manifestation of a similar response to cervical pain in these two calves. Nuchal rigidity was a clinical finding in a report involving ehrlichiosis in children (Bryant \& Marshall, 2000), which was comparable to observations made in our experimental 
calves. In another case report of HME, a patient had a stiff neck as one of the presenting signs upon admission to a hospital (Marty et al., 1995).

Among calves that survived the experimental inoculation with E. chaffeensis, A37, which was inoculated with 91HE17, also experienced a drop in body temperature followed by recumbency at 16 d.p.i. However, this calf recovered after supportive therapy, without antibiotics, and remained in the study. A37 had moderate drops in peripheral platelet and WBC values that reached their lowest points at 14 and 35 d.p.i., respectively, and this calf had a fever that peaked at $39.6{ }^{\circ} \mathrm{C}$ at 25 d.p.i. A35, which was injected with the St Vincent isolate, did not have fever nor did it experience a significant reduction in body temperature. For A35, reductions in platelets and leukocytes were greatest at 17 and 23 d.p.i., respectively. A34, which was injected with the Arkansas strain, displayed a drop in platelets at 1 d.p.i. and increases in both platelets and leukocytes at 37 d.p.i.

The two calves injected with non-infected DH82 cells, A31 and A32, did not display any remarkable clinical signs. A31 had some variation in peripheral leukocyte and platelet levels, but these counts remained within $3 \mathrm{SD}$ of their respective baseline means.

IFA. All calves inoculated with E. chaffeensis-infected DH82 cells seroconverted, except for A36, which died at 6 d.p.i. (Table 3). Calf A38, inoculated with 91HE17, had the highest titre among the calves that died (128 at 7 d.p.i.). Calf A37, which survived exposure to 91HE17, had an initial titre of 32 (7 d.p.i.) that increased later to 128 . A35, which survived exposure to the St Vincent strain, developed the highest antibody titre of 512 (35 d.p.i.). These values were lower than examples reported among experimentally infected dogs, which peaked from 1500 (Dawson \& Ewing, 1992) to 16384 (Zhang et al., 2003), and white-tailed deer, which reached titres of $\sim 1000$

Table 3. Weekly IFA titres of plasma from calves inoculated with active cultures of different $E$. chaffeensis strains

\begin{tabular}{|lllllllllr|}
\hline \multirow{2}{*}{ Inoculum } & Calf & \multicolumn{8}{c|}{ d.p.i. } \\
\cline { 3 - 9 } & & $\mathbf{0}$ & $\mathbf{7}$ & $\mathbf{1 4}$ & $\mathbf{2 1}$ & $\mathbf{2 8}$ & $\mathbf{3 5}$ & $\mathbf{4 2}$ \\
\hline \multirow{2}{*}{ DH82* } & A31 & $-\dagger$ & - & - & - & - & - & - \\
& A32 & - & - & - & - & - & - & - \\
Arkansas & A33 & - & 64 & NA & NA & NA & NA & NA \\
& A34 & - & 64 & 32 & 64 & 128 & 128 & 128 \\
St Vincent & A35 & - & 64 & 64 & 256 & 256 & 512 & 512 \\
& A36 & - & NA & NA & NA & NA & NA & NA \\
91HE17 & A37 & - & 32 & 64 & 128 & 128 & 128 & 64 \\
& A38 & - & 128 & NA & NA & NA & NA & NA \\
& & & & & & & & \\
\hline
\end{tabular}

${ }^{*}$ Negative controls injected with uninfected host cells.

$\uparrow$ Negative at $1: 20$ dilution.

\$NA, Not applicable due to calf mortality.
(Varela et al., 2003). Notably, IFA titres ranging from 64 to 2048 have been reported for diagnosed cases of HME (Yu et al., 1999b). However, these comparisons should be interpreted with caution due to differences among host species, means of host exposure and IFA methods.

PCR assay of peripheral blood. In contrast to the first experiment, buffy coats prepared from all surviving calves inoculated with active cultures tested PCR-positive on multiple occasions (Table 4). Three calves tested positive as early as 5 d.p.i., and a fourth calf (A34) first tested positive at 10 d.p.i. Buffy coats from only one of the deceased calves (A33) tested positive, which was at 3 d.p.i. Calves A34, A35 and A37 tested positive on five, seven and six different dates, respectively. The surviving calves that initially tested PCR-positive subsequently tested negative, and then all of them became positive again before the end of the experiment, which was reminiscent of cyclic rickettsaemias observed for the type species of the family Anaplasmataceae, Anaplasma marginale (Kieser et al., 1990; French et al., 1998).

Xenodiagnosis. Although E. chaffeensis was detected in peripheral blood from all of these calves, the pathogen was detected in fewer ticks than in the first experiment, where most peripheral blood samples were PCR-negative $(P<0.05$, two-tailed Student's $t$ test) (Table 5). Although A35 had the greatest IFA titre, and blood from this calf tested PCR-positive during the nymph acquisition-feeding period, E. chaffeensis was not detected in male ticks of either species that fed as nymphs on this calf.

Xenodiagnosis, the detection of a parasite through biological amplification in a previously unexposed host, has been used for decades to diagnose other infections that are difficult to detect. Accurate detection of E. chaffeensis in

Table 4. PCR results for buffy coats from calves inoculated with active cultures

\begin{tabular}{|c|c|c|c|c|c|c|c|c|c|c|c|c|}
\hline \multirow[t]{2}{*}{ Inoculum } & \multirow[t]{2}{*}{ Calf } & \multicolumn{11}{|c|}{ d.p.i. } \\
\hline & & 0 & 3 & 5 & 7 & 10 & 14 & 17 & 24 & 28 & 35 & 3842 \\
\hline \multirow[t]{2}{*}{ DH82* } & A31 & $-\dagger$ & - & $\mathrm{NT} \ddagger$ & - & - & - & - & - & - & - & -- \\
\hline & A32 & - & - & NT & - & - & - & - & - & - & - & -- \\
\hline \multirow[t]{2}{*}{ Arkansas } & A33 & - & $+\S$ & NAll & NA & NA & NA & NA & NA & NA & NA & NA NA \\
\hline & A34 & - & - & - & - & + & + & + & - & - & - & -+ \\
\hline \multirow[t]{2}{*}{ St Vincent } & A35 & - & - & + & - & - & + & + & + & + & + & -+ \\
\hline & A36 & - & - & - & NA & NA & NA & NA & NA & NA & NA & NA NA \\
\hline \multirow[t]{2}{*}{ 91HE17 } & A37 & - & - & + & + & - & - & - & + & + & + & +- \\
\hline & A38 & - & - & - & - & - & NA & NA & NA & NA & NA & $\mathrm{NA} N \mathrm{NA}$ \\
\hline
\end{tabular}

${ }^{*}$ Control animals inoculated with uninfected DH82 cells.

$\dagger-$, Negative: pathogen not detected.

末NT, Not tested.

$\S+$, Positive: pathogen detected.

IINA, Not applicable due to calf mortality. 
Table 5. PCR-positive male ticks that fed as nymphs on calves inoculated with active cultures (from a total of 10 per host)

\begin{tabular}{|lccc|}
\hline Inoculum & Calf & \multicolumn{2}{c|}{ Tick species } \\
\cline { 3 - 4 } & & $\begin{array}{c}\text { A. americanum } \\
(\boldsymbol{n}=\mathbf{1 0})\end{array}$ & $\begin{array}{c}\text { D. variabilis } \\
(\boldsymbol{n}=\mathbf{1 0})\end{array}$ \\
\hline DH82 & A31 & $-*$ & - \\
& A32 & - & - \\
Arkansas & A34 & 2 & 1 \\
St Vincent & A35 & - & - \\
91HE17 & A37 & - & 3 \\
\hline
\end{tabular}

${ }^{\star}-$, None detected.

subclinical vertebrate hosts can be a substantial challenge. This difficulty was underscored by failure to detect the pathogen in all but two buffy coat samples from calves in the first experiment. Detection of E. chaffeensis in experimentally fed ticks supported other reports of this approach to detect E. chaffeensis and E. canis (Felek et al., 2001; Unver et al., 2001; Loftis et al., 2004; Bremer et al., 2005). Although xenodiagnosis was the most promising approach to detect E. chaffeensis in the first experiment, this procedure resulted in unexpectedly low acquisition rates among ticks fed on calves that received active cultures in the second experiment. Further work is needed to determine whether the means of vertebrate host infection can affect tick infection rates. Both $A$. americanum and $D$. variabilis acquired all three E. chaffeensis strains, confirming other reports that these species acquire this pathogen in nature (Eng et al., 1990a; Anderson et al., 1993; Ewing et al., 1995; Roland et al., 1998; Kramer et al., 1999).

One question regards the apparently paradoxical lack of reported cases of bovine ehrlichiosis, despite their susceptibility to i.v. infection with cultured E. chaffeensis. However, if cattle at risk of exposure to E. chaffeensis are in the range of the primary vector, A. americanum, it is plausible that this range overlaps areas in the US where bovine anaplasmosis is enzootic. Chlortetracycline is often used in these areas as feed supplementation and in saltmineral blocks and mixes to promote food animal production and to control bovine anaplasmosis (Richey et al., 1977; Richey, 1981). Thus, it is tempting to speculate that these subtherapeutic tetracycline rations may also suppress the observation of ehrlichiosis among naturally exposed livestock. Notably, medicated feed was avoided in the present study. Future studies should include evaluation of the efficacy of subtherapeutic tetracycline levels for the prevention of E. chaffeensis infection and ehrlichiosis among naturally and experimentally exposed bovine hosts.

In summary, we now have evidence that calves are susceptible to E. chaffeensis infection, and that the inoculum can influence the clinical outcome of these infections. These infections were confirmed by detection of
E. chaffeensis in peripheral blood and through xenodiagnosis with ticks that acquired the pathogen as nymphs and remained infected through their moult into adults. Furthermore, the cyclic rickettsaemia observed in the second experiment suggested the establishment of an infectious cycle in these hosts. We did not observe differences directly attributable to the different E. chaffeensis strains used for this study, but experimental infections with $91 \mathrm{HE} 17$ appeared more consistently associated with ehrlichiosis as reported for other mammals, while the Arkansas isolate appeared to infect ticks more consistently. The higher doses of active cultures resulted in the most severe disease, suggesting that the bovine model is promising for understanding the immunity and pathogenesis associated with infection by E. chaffeensis. Further work is warranted to optimize the inoculum for this infection model and to determine whether a bovine model would be useful to evaluate factors responsible for the exacerbation of HME, as well as prophylactic and therapeutic approaches to the alleviation of life-threatening forms of this disease.

\section{ACKNOWLEDGEMENTS}

The authors thank Dr Nahad Al-Khalidi, Ashley N. Ankney, Danarra L. Baker, Blair A. Byrd, Erin B. Ervine, Brian W. Martin, Anita R. Miller, Mollie N. Neil, Jaqueline M. Nolting, Courtney L. Raukar, Shweta N. Rao, Julia M. Richardson and Michael B. Waits for technical assistance during this study. This work was supported by NIH grant AI47932.

\section{REFERENCES}

Anderson, B. E., Sims, K. G., Olson, J. G., Childs, J. E., Piesman, J. F., Happ, C. M., Maupin, G. O. \& Johnson, B. J. (1993). Amblyomma americanum: a potential vector of human ehrlichiosis. Am J Trop Med Hyg 49, 239-244.

Barnard, D. R. (1988). Seasonal changes in feeding on cattle and reproduction by Amblyomma americanum (Acari: Ixodidae) under field conditions. J Med Entomol 25, 20-25.

Bishopp, F. C. \& Tremblet, H. L. (1945). Distribution and hosts of certain North American ticks. J Parasitol 31, 1-54.

Bremer, W. G., Schaefer, J. J., Wagner, E. R., Ewing, S. A., Rikihisa, Y., Needham, G. R., Jittapalapong, S., Moore, D. L. \& Stich, R. W. (2005). Transstadial and intrastadial experimental transmission of Ehrlichia canis by male Rhipicephalus sanguineus. Vet Parasitol 131, 95-105.

Bryant, K. A. \& Marshall, G. S. (2000). Clinical manifestations of tickborne infections in children. Clin Diagn Lab Immunol 7, 523-527.

Cheng, C., Paddock, C. D. \& Reddy Ganta, R. (2003). Molecular heterogeneity of Ehrlichia chaffeensis isolates determined by sequence analysis of the 28-kilodalton outer membrane protein genes and other regions of the genome. Infect Immun 71, 187-195.

Davidson, W. R., Lockhart, J. M., Stallknecht, D. E. \& Howerth, E. W. (1999). Susceptibility of red and gray foxes to infection by Ehrlichia chaffeensis. J Wildl Dis 35, 696-702.

Davidson, W. R., Lockhart, J. M., Stallknecht, D. E., Howerth, E. W., Dawson, J. E. \& Rechav, Y. (2001). Persistent Ehrlichia chaffeensis infection in white-tailed deer. J Wildl Dis 37, 538-546. 
Dawson, J. E. \& Ewing, S. A. (1992). Susceptibility of dogs to infection with Ehrlichia chaffeensis, causative agent of human ehrlichiosis. Am J Vet Res 53, 1322-1327.

Dawson, J. E., Stallknecht, D. E., Howerth, E. W., Warner, C., Biggie, K., Davidson, W. R., Lockhart, J. M., Nettles, V. F., Olson, J. G. \& Childs, J. E. (1994). Susceptibility of white-tailed deer (Odocoileus virginianus) to infection with Ehrlichia chaffeensis, the etiologic agent of human ehrlichiosis. J Clin Microbiol 32, 2725-2728.

Dawson, J. E., Biggie, K. L., Warner, C. K., Cookson, K., Jenkins, S., Levine, J. F. \& Olson, J. G. (1996). Polymerase chain reaction evidence of Ehrlichia chaffeensis, an etiologic agent of human ehrlichiosis, in dogs from southeast Virginia. Am J Vet Res 57, 1175-1179.

De Kock, C. \& Quinlan, J. (1926). Splenectomy in Domesticated Animals and its Sequelae with Special Reference to Anaplasmosis in Sheep, 11th and 12th Reports, pp. 369-480. Director of Veterinary Education and Research, Union of South Africa.

Demma, L. J., Holman, R. C., McQuiston, J. H., Krebs, J. W. \& Swerdlow, D. L. (2005). Epidemiology of human ehrlichiosis and anaplasmosis in the United States, 2001-2002. Am J Trop Med Hyg 73, 400-409.

Dugan, V. G., Little, S. E., Stallknecht, D. E. \& Beall, A. D. (2000). Natural infection of domestic goats with Ehrlichia chaffeensis. J Clin Microbiol 38, 448-449.

Dugan, V. G., Varela, A. S., Stallknecht, D. E., Hurd, C. C. \& Little, S. E. (2004). Attempted experimental infection of domestic goats with Ehrlichia chaffeensis. Vector Borne Zoonotic Dis 4, 131-136.

Ellis, E. F. \& Smith, R. T. (1966). The role of the spleen in immunity. With special reference to the post-splenectomy problem in infants. Pediatrics 37, 111-119.

Eng, T. R., Fishbein, D. B., Dawson, J. E., Greene, C. R. \& Redus, M. (1990a). Surveillance of human ehrlichiosis in the United States: 1988. Ann N Y Acad Sci 590, 306-307.

Eng, T. R., Harkess, J. R., Fishbein, D. B., Dawson, J. E., Greene, C. N., Redus, M. A. \& Satalowich, F. T. (1990b). Epidemiologic, clinical and laboratory findings of human ehrlichiosis in the United States, 1988. JAMA ( $\mathrm{A}$ Am Med Assoc) 264, 2251-2258.

Everett, E. D., Evans, K. A., Henry, R. B. \& McDonald, G. (1994). Human ehrlichiosis in adults after tick exposure. Diagnosis using polymerase chain reaction. Ann Intern Med 120, 730-735.

Ewing, S. A., Dawson, J. E., Kocan, A. A., Barker, R. W., Warner, C. K., Panciera, R. J., Fox, J. C., Kocan, K. M. \& Blouin, E. F. (1995). Experimental transmission of Ehrlichia chaffeensis (Rickettsiales: Ehrlichieae) among white-tailed deer by Amblyomma americanum (Acari: Ixodidae). J Med Entomol 32, 368-374.

Felek, S., Unver, A., Stich, R. W. \& Rikihisa, Y. (2001). Sensitive detection of Ehrlichia chaffeensis in cell culture, blood and tick specimens by reverse transcription-PCR. J Clin Microbiol 39, 460-463.

Fishbein, D. B., Dawson, J. E. \& Robinson, L. E. (1994). Human ehrlichiosis in the United States, 1985 to 1990. Ann Intern Med 120, 736-743.

French, D. M., McElwain, T. F., McGuire, T. C. \& Palmer, G. H. (1998). Expression of Anaplasma marginale major surface protein 2 variants during persistent cyclic rickettsemia. Infect Immun 66, 1200-1207.

Garnham, P. C. (1970). The role of the spleen in protozoal infections with special reference to splenectomy. Acta Trop 27, 1-14.

Harrus, S., Waner, T., Keysary, A., Aroch, I., Voet, H. \& Bark, H. (1998). Investigation of splenic functions in canine monocytic ehrlichiosis. Vet Immunol Immunopathol 62, 15-27.

Jones, E. W. \& Brock, W. E. (1966). Bovine anaplasmosis: its diagnosis, treatment and control. J Am Vet Med Assoc 149, 1624-1633.
Kieser, S. T., Eriks, I. S. \& Palmer, G. H. (1990). Cyclic rickettsemia during persistent Anaplasma marginale infection of cattle. Infect Immun 58, 1117-1119.

Kollars, T. M., Jr, Oliver, J. H., Jr, Durden, L. A. \& Kollars, P. G. (2000). Host association and seasonal activity of Amblyomma americanum (Acari: Ixodidae) in Missouri. J Parasitol 86, 1156-1159.

Kramer, V. L., Randolph, M. P., Hui, L. T., Irwin, W. E., Gutierrez, A. G. \& Vugia, D. J. (1999). Detection of the agents of human ehrlichioses in ixodid ticks from California. Am J Trop Med Hyg 60, 62-65.

Lancaster, J. L. (1973). A Guide to the Ticks of Arkansas. University of Arkansas Agricultural Experiment Station Bulletin 779.

Lockhart, J. M. \& Davidson, W. R. (1999). Evaluation of $\mathrm{C} 3 \mathrm{H} / \mathrm{HeJ}$ mice for xenodiagnosis of infection with Ehrlichia chaffeensis. $J$ Vet Diagn Invest 11, 55-59.

Loftis, A. D., Massung, R. F. \& Levin, M. L. (2003). Quantitative realtime PCR assay for detection of Ehrlichia chaffeensis. J Clin Microbiol 41, 3870-3872.

Loftis, A. D., Nicholson, W. L. \& Levin, M. L. (2004). Evaluation of immunocompetent and immunocompromised mice (Mus musculus) for infection with Ehrlichia chaffeensis and transmission to Amblyomma americanum ticks. Vector Borne Zoonotic Dis 4, 323-333.

Marty, A. M., Dumler, J. S., Imes, G., Brusman, H. P., Smrkovski, L. L. \& Frisman, D. M. (1995). Ehrlichiosis mimicking thrombotic thrombocytopenic purpura. Case report and pathological correlation. Hum Pathol 26, 920-925.

Meinkoth, J. H., Ewing, S. A., Cowell, R. L., Dawson, J. E., Warner, C. K., Mathew, J. S., Bowles, M., Thiessen, A. E., Panciera, R. J. \& Fox, C. (1998). Morphologic and molecular evidence of a dual species ehrlichial infection in a dog presenting with inflammatory central nervous system disease. J Vet Intern Med 12, 389-393.

Olano, J. P., Masters, E., Hogrefe, W. \& Walker, D. H. (2003a). Human monocytotropic ehrlichiosis, Missouri. Emerg Infect Dis 9, 1579-1586. Olano, J. P., Hogrefe, W., Seaton, B. \& Walker, D. H. (2003b). Clinical manifestations, epidemiology, and laboratory diagnosis of human monocytotropic ehrlichiosis in a commercial laboratory setting. Clin Diagn Lab Immunol 10, 891-896.

Paddock, C. D., Folk, S. M., Shore, G. M., Machado, L. J., Huycke, M. M., Slater, L. N., Liddell, A. M., Buller, R. S., Storch, G. A. \& other authors (2001). Infections with Ehrlichia chaffeensis and Ehrlichia ewingii in persons coinfected with human immunodeficiency virus. Clin Infect Dis 33, 1586-1594.

Richey, E. J. (1981). Management for anaplasmosis in beef cattle, In Proceedings of The Seventh National Anaplasmosis Conference, Starkville, Mississippi, pp. 567-575.

Richey, E. J., Brock, W. E., Kliewer, I. O. \& Jones, E. W. (1977). Low levels of chlortetracycline for anaplasmosis. Am J Vet Res 38, 171-172.

Rikihisa, Y., Stills, H. \& Zimmerman, G. (1991). Isolation and continuous culture of Neorickettsia helminthoeca in a macrophage cell line. J Clin Microbiol 29, 1928-1933.

Rikihisa, Y., Ewing, S. A. \& Fox, J. C. (1994). Western immunoblot analysis of Ehrlichia chaffeensis, E. canis, or E. ewingii infections in dogs and humans. J Clin Microbiol 32, 2107-2112.

Roby, T. O., Gates, G. W. \& Mott, L. O. (1961). The comparative susceptibility of calves and adult cattle to bovine anaplasmosis. Am J Vet Res 22, 982-985.

Roland, W. E., McDonald, G., Caldwell, C. W. \& Everett, E. D. (1995). Ehrlichiosis - a cause of prolonged fever. Clin Infect Dis 20, 821-825.

Roland, W. E., Everett, E. D., Cyr, T. L., Hasan, S. Z., Dommaraju, C. B. \& McDonald, G. A. (1998). Ehrlichia chaffeensis in Missouri ticks. Am J Trop Med Hyg 59, 641-643. 
Smith, C. N., Cole, M. M. \& Gouk, H. K. (1946). Biology and Control of the American Dog Tick. USDA Technical Bulletin TB0905.

Stich, R. W., Kocan, K. M., Palmer, G. H., Ewing, S. A., Hair, J. A. \& Barron, S. J. (1989). Transstadial and attempted transovarial transmission of Anaplasma marginale by Dermacentor variabilis. Am $J$ Vet Res 50, 1377-1380.

Stone, J. H., Dierberg, K., Aram, G. \& Dumler, J. S. (2004). Human monocytic ehrlichiosis. JAMA (J Am Med Assoc) 292, 2263-2270.

Unver, A., Ohashi, N., Tajima, T., Stich, R. W., Grover, D. \& Rikihisa, Y. (2001). Transcriptional analysis of $p 30$ major outer membrane multigene family of Ehrlichia canis in dogs, ticks, and cell culture at different temperatures. Infect Immun 69, 6172-6178.

Unver, A., Rikihisa, Y., Stich, R. W., Ohashi, N. \& Felek, S. (2002). The omp-1 major outer membrane multigene family of Ehrlichia chaffeensis is differentially expressed in canine and tick hosts. Infect Immun 70, 4701-4704.

Varela, A. S., Stallknecht, D. E., Yabsley, M. J., Moore, V. A., Davidson, W. R. \& Little, S. E. (2003). Experimental infection of white-tailed deer (Odocoileus virginianus) with Ehrlichia chaffeensis by different inoculation routes. J Wildl Dis 39, 881-886.

Wagner, E. R., Bremer, W. G., Rikihisa, Y., Ewing, S. A., Needham, G. R., Unver, A., Wang, X. \& Stich, R. W. (2004). Development of a p28-based PCR assay for Ehrlichia chaffeensis. Mol Cell Probes 18, 111-116.

Wen, B., Rikihisa, Y., Mott, J. M., Greene, R., Kim, H. Y., Zhi, N., Couto, G. C., Unver, A. \& Bartsch, R. (1997). Comparison of nested PCR with immunofluorescent-antibody assay for detection of Ehrlichia canis infection in dogs treated with doxycycline. J Clin Microbiol 35, $1852-1855$.

Williams, C. V., Van Steenhouse, J. L., Bradley, J. M., Hancock, S. I., Hegarty, B. C. \& Breitschwerdt, E. B. (2002). Naturally occurring Ehrlichia chaffeensis infection in two prosimian primate species: ringtailed lemurs (Lemur catta) and ruffed lemurs (Varecia variegata). Emerg Infect Dis 8, 1497-1500.

Yu, X. J., McBride, J. W. \& Walker, D. H. (1999a). Genetic diversity of the 28-kilodalton outer membrane protein gene in human isolates of Ehrlichia chaffeensis. J Clin Microbiol 37, 1137-1143.

Yu, X. J., Crocquet-Valdes, P. A., Cullman, L. C., Popov, V. L. \& Walker, D. H. (1999b). Comparison of Ehrlichia chaffeensis recombinant proteins for serologic diagnosis of human monocytotropic ehrlichiosis. J Clin Microbiol 37, 2568-2575.

Zhang, X. F., Zhang, J. Z., Long, S. W., Ruble, R. P. \& Yu, X. J. (2003). Experimental Ehrlichia chaffeensis infection in beagles. $J$ Med Microbiol 52, 1021-1026. 